\title{
CalciumCV: Computer vision software for calcium signaling in astrocytes ${ }^{\star}$
}

\author{
V. Kustikova ${ }^{1}$, M. Krivonosov ${ }^{1}$, A. Pimashkin ${ }^{1}$, P. Denisov $^{1}$, A. Zaikin ${ }^{1,2}$, \\ M. Ivanchenko ${ }^{1}$, I. Meyerov ${ }^{1 凶}$, and A. Semyanov ${ }^{1,3 凶}$ \\ 1 Lobachevsky State University of Nizhni Novgorod, Nizhni Novgorod, 603950, \\ Russian Federation, \\ 2 Department of Mathematics and Institute for Women's Health, University College \\ London, London WC1E 6AU, United Kingdom \\ 3 Shemyakin-Ovchinnikov Institute of bioorganic chemistry, Moscow, 117997, \\ Russian Federation, \\ valentina.kustikova@itmm.unn.ru, mike_live@mail.ru, \\ pimashkin@neuro.nnov.ru, denisov@neuro.nnov.ru, alexey.zaikin@ucl.ac.uk, \\ ivanchenko.mv@gmail.com, meerov@vmk.unn.ru, semyanov@neuro.nnov.ru
}

\begin{abstract}
Developing computational analysis of time-lapse imaging of calcium events in astrocytes is a challenging task in neuroscience. Here we report the implementation of an algorithm that solves this task. After noise reduction with the block-matching and 3D filtering (BM3D) algorithm, calcium activity is identified as fluorescence elevation above the baseline level. Individual events are detected by sliding window approach applied to the variation of pixel intensity relative to the baseline level. The maximal projection and duration of astrocytic calcium events are then assessed. The novelty of the proposed method is an adaptive construction of the baseline level. The statistical results generated by our program are consistent with the previous algorithm reported and used by us for the reference. The software is publicly available.
\end{abstract}

Keywords: bioinformatics, calcium signaling in astrocytes, computer vision, image analysis, statistical analysis

\section{Introduction}

Astrocytes are electrically inactive brain cells, which are connected through gap-junctions and form a syncytium-like functional network [1,2. Although the mechanisms of astrocytic $\mathrm{Ca}^{2+}$ dynamics have been studied for more than quarter of a century, assessment of spatial-temporal properties of individual $\mathrm{Ca}^{2+}$ events in the entire astrocyte remains a challenging task.

Imaging of calcium activity is a basic method for calcium activity analysis. The simplest approach to this problem is an analysis of $\mathrm{Ca}^{2+}$ dynamics

* This research financially supported by Russian Science Foundation (AZ to 16-1200077, algorithm; AS to 16-14-00201, data analysis). 
in adjustment regions of interest (ROI) $\sqrt[3]{5}$ in two-dimensional plane. For example, if an event starts on one ROI and propagates to the neighboring ROIs, one can estimate its spread by counting the number of involved ROIs. However, this method is laborious and provides less accurate information about size and duration of $\mathrm{Ca}^{2+}$ events than measuring their actual contours in each imaging frame. Recently, several approaches to measure automatically the parameters of individual $\mathrm{Ca}^{2+}$ events in time-lapse imaging data have been suggested 68 , however, none of these methods has become a standard. Nowadays, advanced methods of three-dimensional $\mathrm{Ca}^{2+}$ imaging are developed [9, but application of these methods requires the appropriate hardware for the experiments that is not widespread.

The state-of-the-art approaches for image preprocessing and solving computer vision problems are based on deep learning, especially, on convolutional neural networks 10 15. Application of deep learning supposes the existence of a large train dataset. During astrocyte activity analysis, to get new data means to carry out new biological experiment which requires corresponding materials and hardware. The labeling data is performed by experts, but there are astrocyte activities which may be invisible for some of them that is why the labeling is a subjective process. Therefore, the application of deep learning to the astrocyte activity analysis is complicated by the high-level complexity of data preparation.

We improved the previously developed method 16 to analyse time lapse imaging records for the calcium activity of an astrocyte. To do this, we made two main modifications in the event detection flow. First, we implemented a new precise and adaptive algorithm for a baseline level approximation to detect the inactive state of the astrocyte. Second, we proposed using the sliding window approach to detect calcium events. We show that the algorithm detects $\mathrm{Ca}^{2+}$ events in imaging data and extracts their duration and maximal projection. The new implementaion improves performance by $16-40 \%$. The results are in good agreement with the previous report 16 .

\section{Calcium Event Detection}

\subsection{Problem Statement}

The proposed method analyses time lapse imaging records for the calcium activity of an astrocyte. Each frame displays a spatial distribution of fluorescence intensity in several planes parallel to the substrate, yielding a set of planar images that reflect calcium activity. Further on, we introduce and consider an instantaneous maximal projection, defined as the maximal intensity at each point over this set (Fig. 1):

$$
V=\left\{V_{s}, 0 \leq s \leq k-1\right\}, V_{s}=\left(V_{i j}^{(s)}: 0 \leq i \leq h-1,0 \leq j \leq w-1\right),
$$

where $k$ is a number of frames, and $V_{s}$ is a frame with a resolution $w \times h$.

Moreover, we have a movie

$$
N=\left\{N_{r}, 0 \leq r \leq n-1\right\}, N_{r}=\left(N_{i j}^{(r)}: 0 \leq i \leq h-1,0 \leq j \leq w-1\right),
$$




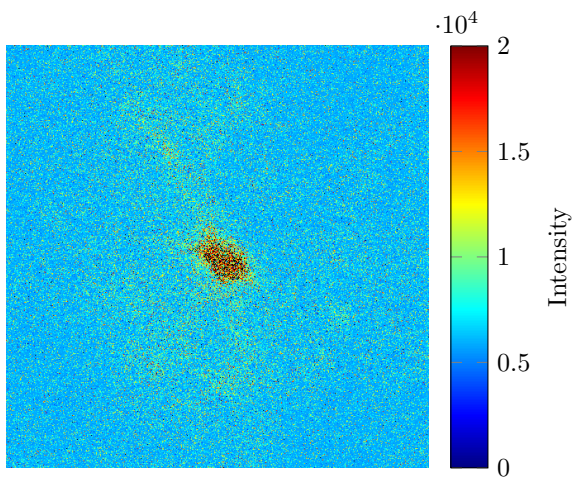

Fig. 1. The instantaneous maximal projection of the fluorescence intensity over a set of planar sections for an astrocyte. Shifting from the blue to red end of the color map codes increasing brightness, units are arbitrary (color online).

that corresponds to the camera noise (Fig. 2) and represents a video without astrocyte. Here, $n$ is a number of noise video frames, and $N_{r}$ is a noise frame. The principle of noise generation on videos is unknown; the noise model is Gaussian noise.

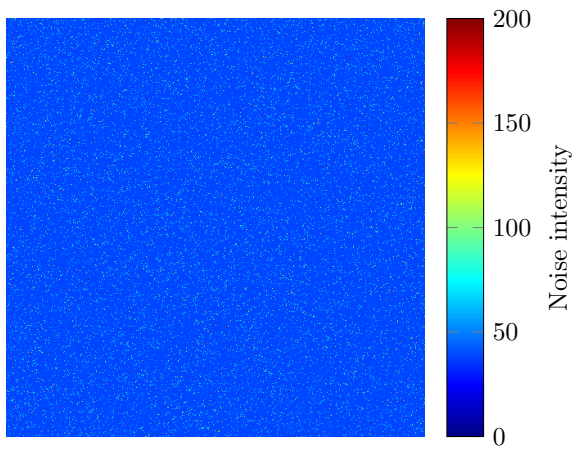

Fig. 2. The noise frame sample. Shifting from the blue to red end of the color map codes increasing brightness, units are arbitrary (color online).

The proposed method processes time lapse imaging records and noise records, and extracts a set of calcium events in the astrocyte. A single event is assigned with start and end times (frame identifiers), and described by a set of points (frame pixels) that belong to it over the whole time span (a set of frames). For the extracted events the statistical analysis is carried out. We analyse the statistical properties of event time spans and maximal projections. 


\subsection{Method}

General Pipeline. The developed method includes the following steps (Fig. 3):

1. Video preprocessing:

- Aligning an image (eliminating jitter).

- Calculating noise parameters and subtracting the camera noise.

- Filtering video frames.

- Evaluating a noise level on the filtered video.

2. Calculating a baseline of the fluorescence intensity and its relative variation.

3. Detecting calcium events based on the relative intensity variation.

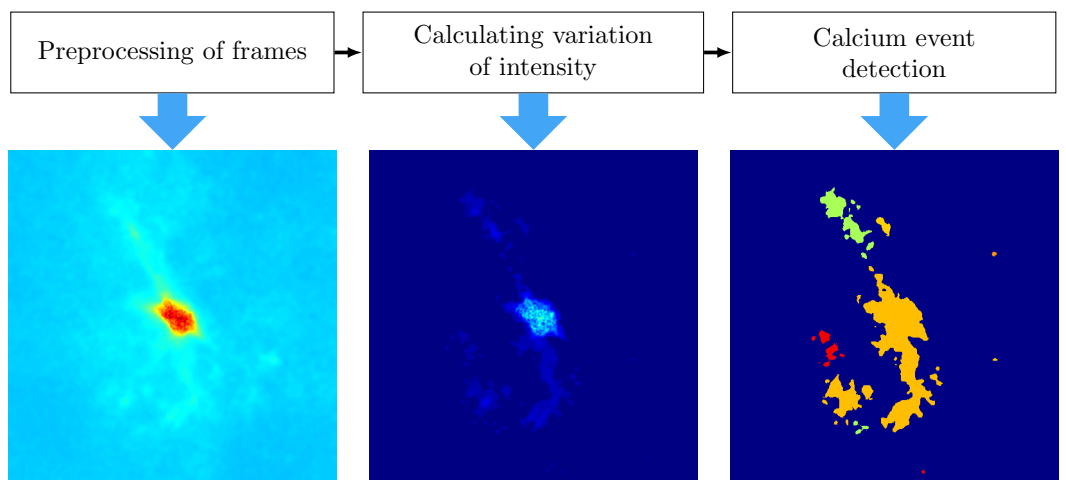

Fig. 3. Pipeline of the proposed method (color online).

Video Preprocessing. This step consists of 4 operations. Let us consider each operation in detail.

1. Aligning an image. To align each video frame $V_{s}$ we compute normalized cross-correlation $C(i, j)$ of the first frame and the current frame shifted by a vector $(i, j)$, where $i=\overline{-w, w}, j=\overline{-h, h}$. The optimal shift should be computed as a decision of the following optimization problem:

$$
\left(i_{\text {opt }}, j_{\text {opt }}\right)=\arg \max _{(i, j)} C(i, j) .
$$

Next, we denote aligned video as $V$.

2. Calculating noise parameters and subtracting the camera noise. To identify noise parameters noise video $N$ should be considered. We calculate mean noise frame by time

$$
\begin{gathered}
E N=\langle N\rangle_{r}=\left(E N_{i j}: 0 \leq i \leq h-1,0 \leq j \leq w-1\right), \\
E N_{i j}=\frac{1}{n} \sum_{r=0}^{n-1} N_{i j}^{(r)}
\end{gathered}
$$


and standard deviation of the noise

$$
\begin{gathered}
S N=\sigma_{r}(N-E N)=\left(S N_{i j}: 0 \leq i \leq h-1,0 \leq j \leq w-1\right), \\
S N_{i j}=\sqrt{\frac{\sum_{r=0}^{n-1}\left(N_{i j}^{(r)}-E N_{i j}\right)^{2}}{n-1}} .
\end{gathered}
$$

Similarly we compute mean frame and standard deviation of the input video:

$$
\begin{gathered}
E V=\langle V\rangle_{s}=\left(E V_{i j}: 0 \leq i \leq h-1,0 \leq j \leq w-1\right), \\
E V_{i j}=\frac{1}{k} \sum_{s=0}^{k-1} V_{i j}^{(s)}, \\
S V=\sigma_{s}(V-E V)=\left(S V_{i j}: 0 \leq i \leq h-1,0 \leq j \leq w-1\right) . \\
S V_{i j}=\sqrt{\frac{\sum_{s=0}^{k-1}\left(V_{i j}^{(s)}-E V_{i j}\right)^{2}}{k-1}} .
\end{gathered}
$$

Pixel $(i, j)$ for which $S V_{i j}<\alpha \cdot S N_{i j}$ is considered as a point of undefined activity ( $\alpha$ is a parameter, default value is 3). Undefined activity means that the point constains camera measurement error or minor changes of the intensity.

Thereafter we need to eliminate camera measurement error by subtracting the mean noise frame of the video frames

$$
\begin{gathered}
O V=\left\{O V_{s}, s=0 \ldots k-1\right\}, O V_{s}=\left(O V_{i j}^{(s)}: 0 \leq i \leq h-1,0 \leq j \leq w-1\right), \\
O V_{i j}^{(s)}=V_{i j}^{(s)}-E N_{i j} .
\end{gathered}
$$

3. Filtering video frames. Filtering video $O V$ using block-matching and $3 \mathrm{D}$ filtering (BM3D) 17] and Gaussian filters allows to smooth video and to prepare it for post-processing. BM3D is based on the idea that real-world images contain similar patches. The similarity of these patches is calculated by $L_{2}$-norm. Patches are grouped, and 3D-transform is applied to the group. The transform output is spectral representation of the block group. Filtering spectral coefficients by internal filter and applying inverse transform allow to restore filtered image. Let us denote the filtered video as $F V$ :

$$
F V=\left\{F V_{s}, 0 \leq s \leq k-1\right\}, F V_{s}=\left(F V_{i j}^{(s)}: 0 \leq i \leq h-1,0 \leq j \leq w-1\right),
$$

where $F V_{s}$ is a filtered video frame. The application of BM3D is explained by the successful experience of its using in 16 . One of the directions for the future research related with the investigation of applying more advanced denoising methods 1821 .

4. Evaluating a noise level on the filtered video. Further we consider pixels with defined activity to evaluate a noise level on the filtered video $F V$. The task is to estimate standard deviation $\sigma_{s}(F V-R V)$ the intensity of the filtered video $F V$ relative to the unknown real signal $R V$, where

$$
R V=\left\{R V_{s}, 0 \leq s \leq k-1\right\}, R V_{s}=\left(R V_{i j}^{(s)}: 0 \leq i \leq h-1,0 \leq j \leq w-1\right) .
$$


We assume that if $S N=\sigma_{r}(N-E N)$ is a standard deviation of the noise video then $S F N=\sigma_{r}(F N-E N)$ is a standard deviation of the filtered noise video $F N$ that described as follows:

$$
\begin{gathered}
F N=\left\{F N_{r}, 0 \leq r \leq n-1\right\}, \\
F N_{r}=\left(F N_{i j}^{(r)}: 0 \leq i \leq h-1,0 \leq j \leq w-1\right) .
\end{gathered}
$$

Therefore, a coefficient of variation of the standard deviation after filtering the noise video can be computed as follows:

$$
R=\left\langle\frac{S N}{S F N}\right\rangle_{i j}=\frac{1}{w \cdot h} \sum_{i=0}^{h-1} \sum_{j=0}^{w-1} \frac{S N_{i j}}{S F N_{i j}} .
$$

Hence, $\sigma_{r}(N-E N) \approx R \cdot \sigma_{r}(F N-E N)$, and we can suppose that

$$
\sigma_{s}(V-R V) \approx R \cdot \sigma_{s}(F V-R V) .
$$

Since real signal $R V$ is unknown and applied filter is good enough (i.e. $F V \approx$ $R V)$, it can be assumed $\sigma_{s}(V-R V) \approx \sigma_{s}(V-F V)$. Consequently, we can estimate standard deviation $\sigma_{s}(F V-R V)$ of the intensity of the filtered video $F V$ relative to the unknown real signal $R V$ as follows:

$$
\sigma=S R=\sigma_{s}(F V-R V) \approx \frac{\sigma_{s}(V-F V)}{R} .
$$

Using this, we classify pixels as noise if intensity variation relative to the baseline level is less than $3 \cdot \sigma$ (Fig. 4).

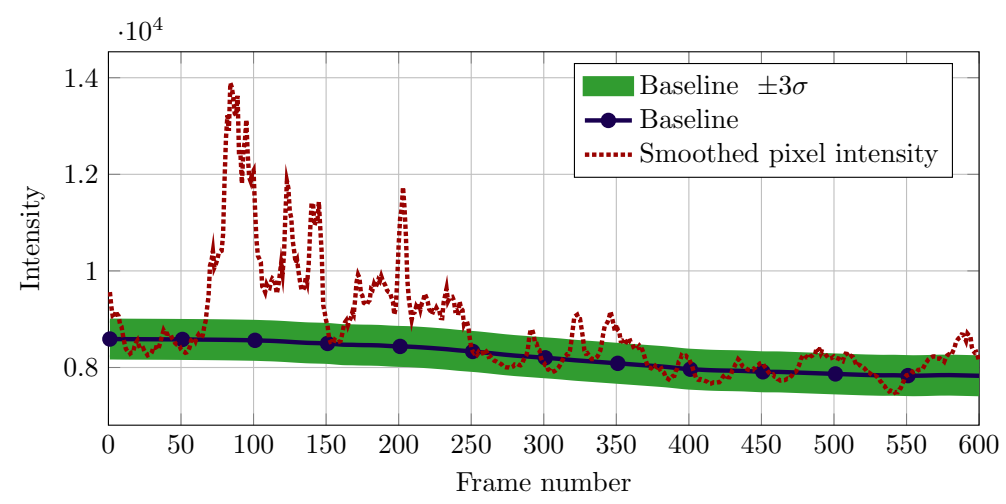

Fig. 4. Baseline pixel intensity and three standard deviations from it (color online). 
Calculating a baseline of the fluorescence intensity and its relative variation. A baseline of the fluorescence intensity corresponds to the inactive state of the astrocyte. Let us introduce some auxiliary notation: $F=\left\{F^{t}, 0 \leq\right.$ $t \leq k-1\}$ is a pixel intensity realization in time, $F_{0}=\left\{F_{0}^{t}, 0 \leq t \leq k-1\right\}$ is a baseline pixel intensity that corresponds to the inactive astrocyte state in the pixel. Calculating the baseline intensity $F_{0}$ for each pixel assumes an iterative approximation by applying moving average for the current estimate of a baseline (Fig. 5). At the first iteration we suppose that the baseline is equal to the pixel intensity $F_{0}=F$. The fixed-size moving average is applied to the intensity realization, and we compute first approximation of the baseline $F_{0}$. The window size is a parameter and its value is choosen based on the video capture parameters. Further, we compute the difference between pixel intensity realization $F$ and current baseline approximation $F_{0}$. If the difference is less than zero then we set it to zero since it is an impossible situation from the point of view of astrocyte activity. Then the standard deviation of such difference is computed. The iterative process stops when this standard deviation is less than the noise level represented earlier. It should be noted that the novelty of the method consists in the iterative procedure of the fluorescence intensity baseline.

The relative intensity variation for each pixel is calculated as follows:

$$
\frac{d F}{F_{0}}=\frac{F-F_{0}}{F_{0}}
$$

This equation is used to compute calcium fluorescence 22].

We classify the pixel as active at the frame number $t$ if $\frac{F^{t}-F_{0}^{t}}{F_{0}^{t}}>0$ otherwise the pixel is considered to be inactive.

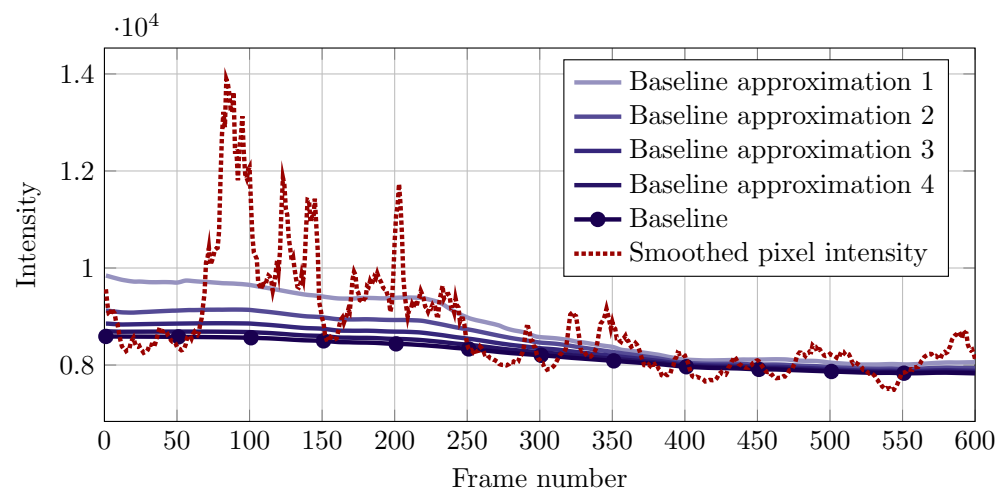

Fig. 5. Iterative approximation of a baseline of the fluorescence intensity (color online).

Detecting calcium events based on the relative intensity variation. First of all we need to identify time gaps where each pixel is active. This step is 
implemented by clustering the activity moments using DBSCAN 23 method. Next, these time gaps are combined over space and time using sliding window approach. The constructed time gaps for the set of all pixels are represented then as a set of graph vertices. Two vertices are connected by an edge if the corresponding pixels belong to the same window location and the corresponding time gaps intersect. We construct connectivity components for this graph, where each connectivity component represents an astrocyte event. Consequently all event points may be reconstructed based on the data stored in the graph vertices.

\section{Implementation}

The proposed method is implemented in $\mathrm{C}++$ programming language, tools for statistical events analysis are implemented in MATLAB. The code is distributed free and open source. The source code can be downloaded from GitHub: https://github.com/UNN-VMK-Software/astro-analysis. We also submit a short test movie and step-by-step tutorial for building and executing developed program, and for fine-turning method parameters.

\section{Experiments}

We tested the developed method on the available experimental data and verified its validity. We used 10 records with a duration from 100 to 3000 frames, with frame resolution is not exceeding $512 \times 512$ pixels (Table 1 ).

Table 1. Test movie parameters.

\begin{tabular}{|c|c|r|r|}
\hline \# & Resolution & Duration & Number of detected events \\
\hline 1 & $512 \times 512$ & 1500 & 465 \\
\hline 2 & $451 \times 441$ & 3000 & 1204 \\
\hline 3 & $421 \times 512$ & 3000 & 2048 \\
\hline 4 & $512 \times 512$ & 100 & 65 \\
\hline 5 & $512 \times 512$ & 1000 & 458 \\
\hline 6 & $512 \times 512$ & 1000 & 591 \\
\hline 7 & $500 \times 390$ & 1000 & 260 \\
\hline 8 & $512 \times 512$ & 200 & 61 \\
\hline 9 & $512 \times 512$ & 500 & 97 \\
\hline 10 & $512 \times 512$ & 600 & 364 \\
\hline
\end{tabular}

The correctness of event identification in a given subject area is determined today by the method of expert assessments. Unfortunately, we do not know the accurate metrics.

Firstly, identification of calcium events was confirmed by visual inspection. Fig. 6 exemplifies the snapshots after filtering, the intensity variation patterns, and the set of detected events. A detailed verification can be done, for exam- 

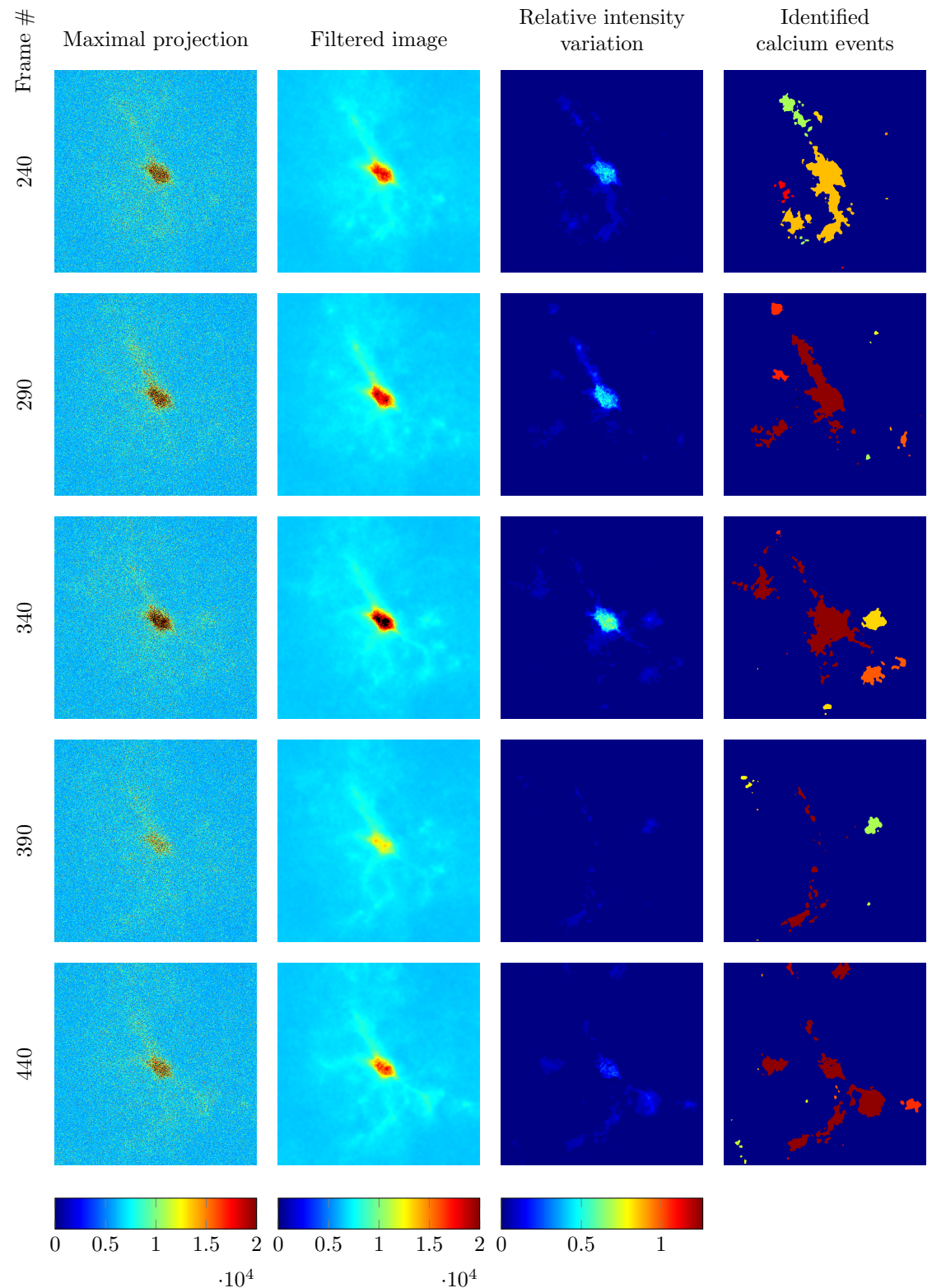

Fig. 6. Intermediate and final results of the astrocyte calcium events detection method for a test movie for the frames from 240 to 440 with the 50 frame step (color online). 
ple, with the prepared movie: https://cloud.mail.ru/public/4Zhh/BCjo7KN86. Clearly, the method adequately yields the regions of calcium activity.

Secondly, we implemented a statistical analysis of calcium events. The complementary cumulative distribution functions for durations and maximal projections of events are approximated well by power laws, as confirmed by KolmogorovSmirnov test. The typical values of the exponents are consistent with the previously reported data (Fig. 7, 8). A comparison with the results of the approach

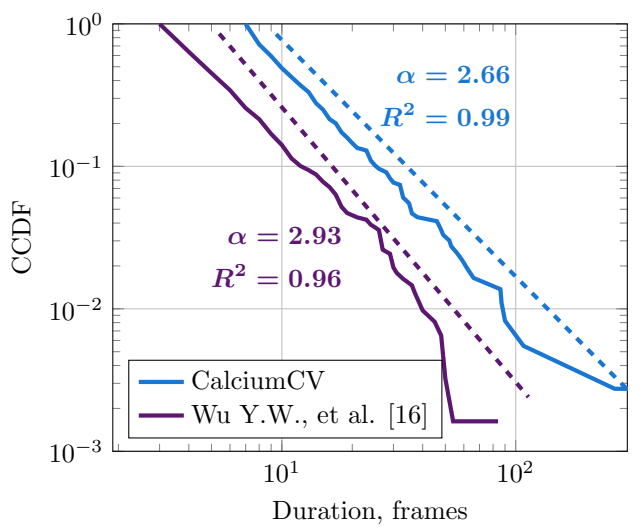

Fig. 7. The complementary cumulative distribution functions (CCDF) for event durations and the power law fits in logarithmic scale: $\alpha$ is a value of the exponent, $R^{2}$ is an approximation accuracy (color online).

described in 16 demonstrates that the novel method allows more accurate baseline approximation, crucial in certain cases. While in [16] it is taken that fluorescence at a pixel belongs to background if its intensity is below a certain threshold, our method employs the adaptive threshold both in time and space. Moreover, parameters of the baseline level are different for different pixels. It tackles the typical pitfalls of global thresholding, such as picking noise for its overly low value and missing actual events when it is set overly high, with inevitable mistakes if the amplitude ranges of noise and events overlap.

Performance of the software has been also tested as following. The total execution time for the sample movie that contains 600 frames with $512 \times 512$ resolution approximately equals 10.39 minutes, that compares to the method from 16 (about 12.38 minutes), given the test computational environment with Intel ${ }^{\circledR}$ Core $^{\mathrm{TM}}$ i7 870, $2.93 \mathrm{GHz}, 4$ cores, $8 \mathrm{~GB}$ RAM, OS Windows (64-bit). The new implementation improves performance by $16 \%$. This measurement value grows to $40 \%$ when processing more complicated movies which contain large number of events. At the same time the implementation of the proposed method was serial, and multi-threaded in case of [16]. Finally, we notice the potential for accelerating the developed software by optimizing and parallelizing some 


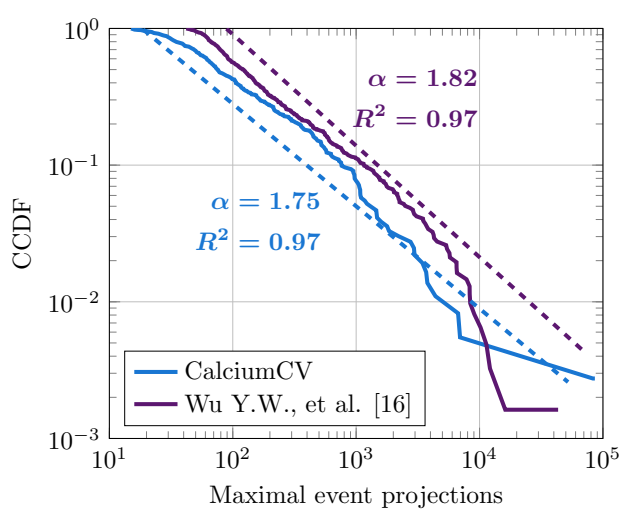

Fig. 8. The complementary cumulative distribution functions (CCDF) for event maximal projections and the power law fits in logarithmic scale: $\alpha$ is a value of the exponent, $R^{2}$ is an approximation accuracy (color online).

method steps. Performance analysis is not the main subject of this research, here we demonstrate that the developed implementation works in a reasonable time.

\section{Conclusion}

In summary, we have demonstrated a method for detecting calcium signalling events in astrocytes. The novelty of the proposed method consists in constructing the baseline level corresponding to the inactive state of astrocyte. Moreover, the analysis of software performance shows that the time of astrocyte event detection for the developed implementation is less than for the existing one. The software is available at GitHuh 4 for testing on raw experimental data. Our program can be used by researchers analyzing spatiotemporal properties of calcium events in astrocytes and other cell types such as calcium sparks in cardiomyocytes. The method is open for future development.

\section{References}

1. Bennett, M.V., et al.: New roles for astrocytes: gap junction hemichannels have something to communicate, Trends Neurosci, 26(11), 610-617 (2003)

2. Ma, B., et al.: Gap junction coupling confers isopotentiality on astrocyte syncytium, Glia, 64(2), 214-226 (2016)

3. Nett, W.J., et al.: Hippocampal astrocytes in situ exhibit calcium oscillations that occur independent of neuronal activity, J Neurophysiol, 87(1), 528-537 (2002)

4. Sun, M.Y., et al.: Astrocyte calcium microdomains are inhibited by Bafilomycin A1 and cannot be replicated by low-level Schaffer collateral stimulation in situ, Cell Calcium, 55(1), 1-16 (2014)

\footnotetext{
4 https://github.com/UNN-VMK-Software/astro-analysis
} 
5. Fiacco, T.A., et al.: Intracellular astrocyte calcium waves in situ increase the frequency of spontaneous AMPA receptor currents in CA1 pyramidal neurons, J Neurosci, 24(3), 722-732 (2004)

6. Asada, A., et al.: Subtle modulation of ongoing calcium dynamics in astrocytic microdomains by sensory inputs, Physiol Rep, 3(10) (2015)

7. Nakayama, R., et al.: Subcellular calcium dynamics during juvenile development in mouse hippocampal astrocytes, Eur J Neurosci, 43(7), 923-932 (2016)

8. Shigetomi, E., et al.: Probing the Complexities of Astrocyte Calcium Signaling, Trends Cell Biol, 26(4), 300-312 (2016)

9. Bindocci, E., et al.: Three-dimensional Ca2+ imaging advances understanding of astrocyte biology, American Association for the Adv. of Science, 356(6339) (2017)

10. Krizhevsky, A., et al.: ImageNet Classification with Deep Convolutional Neural Networks // Adv. in Neural Inf. Proc. Systems 25 (NIPS 2012), 1097-1105 (2012)

11. Szegedy, C., et al.: Going Deeper with Convolutions, http://arxiv.org/abs/ $1409.4842(2014)$

12. Simonyan, K., et al.: Very Deep Convolutional Networks for Large-Scale Visual Recognition, http://www.robots.ox.ac.uk/ vgg/research/very_deep (2014)

13. Redmon, J., et al.: You Only Look Once: Unified, Real-Time Object Detection, https://arxiv.org/abs/1506.02640 (2016)

14. Redmon, J., Farhadi, A.: YOLO9000: Better, Faster, Stronger, https://arxiv. org/abs/1612.08242 (2016)

15. Liu, W., et al.: SSD: Single Shot MultiBox Detector. https://arxiv.org/abs/ 1512.02325 (2016)

16. $\mathrm{Wu}, \mathrm{Y} . \mathrm{W}$., et al.: Spatiotemporal calcium dynamics in single astrocytes and its modulation by neuronal activity, Cell Calcium, 55(2), 119-129 (2014)

17. Dabov, K., et al.: Image denoising by sparse 3d transform domain collaborative filtering, IEEE Transactions on Image Processing, 16(8) (2007)

18. Ji, H., et al.: Robust video denoising using low rank matrix completion, IEEE Comp. Society Conf. on Comp. Vision and Pattern Recognition, 1791-1798 (2010)

19. Burges, C.J.C., et al.: Adaptive Multi-Column Deep Neural Networks with Application to Robust Image Denoising, Advances in Neural Information Processing Systems 26 (NIPS 2013), 1493-1501 (2013)

20. Lefkimmiatis, S.: Non-Local Color Image Denoising with Convolutional Neural Networks, IEEE Int. Conf. on Computer Vision and Pattern Recognition (2017)

21. Lefkimmiatis, S.: Universal Denoising Networks: A Novel CNN Architecture for Image Denoising, IEEE Int. Conf. on CVPR (2017)

22. Kao, J.P.Y., et al.: Photochemically Generated Cytosolic Calcium Pulses and Their Detection by Fluo-3, The J. of Biological Chemistry, 264(14), 8179-8184 (1989)

23. Ester, M., et al.: A Density-Based Algorithm for Discovering Clusters in Large Spatial Databases with Noise, Proc. of the Second Int. Conf. on Knowledge Discovery and Data Mining (KDD-96), 226-231 (1996) 